Phosphorylase-Aktivität wirkt, cAMP kann aber nicht der einzige regulierende Faktor sein, da bei der hohen Reizfrequenz die Phosphorylase-Aktivität sinkt, obwohl die cAMP-Konzentration erhöht ist. Da gleichzeitig eine Erschlaffung der Muskeln eintritt, also eine Abnahme der $\mathrm{Ca}^{2+}$-Konzentration im Sarcoplasma stattgefunden hat, ist zu vermuten, dass der Rückgang der PhosphorylaseAktivität ebenfalls durch die Abnahme der Konzentration an freiem $\mathrm{Ca}^{2+}$ verursacht wird. Das entspricht den erwähnten Untersuchungen von HANSFORD und SACKTOR ${ }^{6}$.

14 Der benutzte Szintillationszähler wurde aus Mitteln des Landesamtes für Forschung, Düsseldorf angeschafft.
Welche Rolle das zyklische AMP in den Flugmuskeln von L. migratoria spielt, ist Gegenstand weiterer Untersuchungen.

Summary. In half-thorax of Locusta migratoria, the activity of phosphorylases and the level of cAMP was studied during electrical stimulation of flight muscles. Both phosphorylase activity and cAMP level increased during phasic and tetanic contractions but no exact correlation could be found.

C. BOCKBREDER ${ }^{14}$

Zoologisches Institut der Universität

Tierphysiologischer Lehrstuhl, Hindenburgplatz 55,

D-44 Münster (BRD), 8 June 1974.

\title{
Transplantation and 'Cross-Transplantation' of Free Muscle Grafts in the Rat ${ }^{1}$
}

The free grafting of entire mammalian skeletal muscles has seldom been employed in either experimental or clinical applications. Free grafts of muscle weighing more than 1 or $2 \mathrm{~g}$ are normally unsuccessful. The transplanted muscle necrotizes and is largely replaced by a band of dense connective tissue. In contrast, small muscles ${ }^{2}$ or larger muscles that have been denervated beforehand ${ }^{3-5}$ can be freely grafted with considerable success.

This investigation was designed to compare the reactions to free autografting of normal and previously denervated extensor digitorum longus (EDL) and soleus (SOL) muscles in young rats. The grafting technique was then used to study the effects of cross-transplanting a fast muscle into the bed of a slow one and vice versa. Earlier morphological work ${ }^{5,6}$ has shown that the basis for the success of freely grafted muscles is the early degeneration of most of the mature muscle fibres within the graft and the subsequent regeneration of a new population of muscle fibres. Therefore the experiments on cross transplantation of fast and slow muscles demonstrate the influence of the nerve supply upon functional properties of regenerating muscle in contrast to previous cross-innervation studies in which the muscle remains anatomically and histologically stable, and

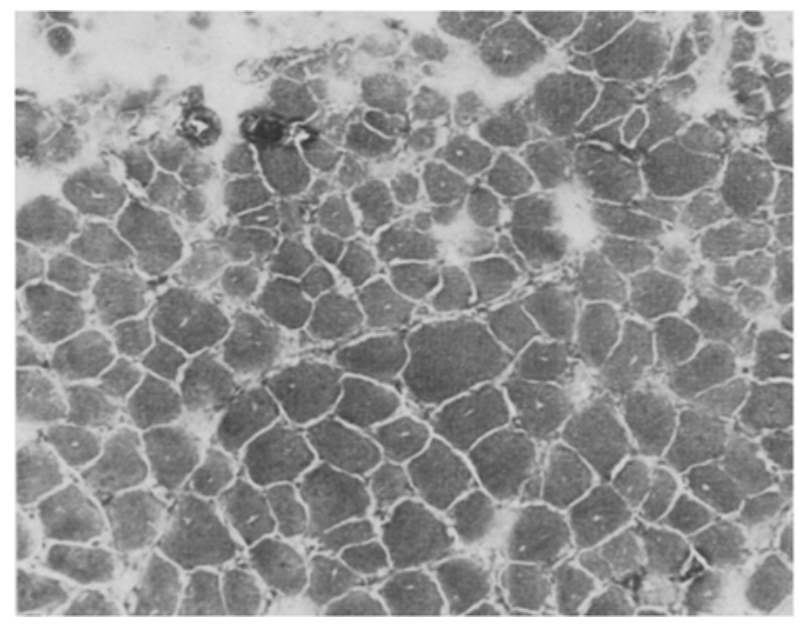

Fig. 1. 30-day extensor digitorum longus transplant stained for ATPase activity. The staining pattern of the muscle fibres is uniform throughout. only its nerve supply is changed. The cross-transplanted muscles are not only innervated by a foreign nerve, but they are also located in the functional environment of the type of muscle in whose place they are substituted. These differences between cross-innervation and cross-transplantation models could be advantageous in analysis of long term nerve-muscle interactions?

Materials and methods. The experiments were carried out upon 1-month old male Wistar rats, $(60 \mathrm{~g})$. Transplantation was done by removing intact the EDL or SOL muscle from one leg and transplanting it in place of the

1 Work performed during an exchange between the Czechoslovak and US Academies of Sciences and supported in part by a grant from the MDAA.

2 R. P. Zhenevskaya, O. N. Rumyantseva, I. L. Novoselova and E. V. Proshlyakova, Zh. obshch. Biol. 26, 569 (1965).

3 A. N. Studitsky and N. N. Bosova, Arkh. Anat. Gistol. Embriol. $39,18(1960)$.

${ }^{4}$ N. Thompson, Plastic reconstr. Surg. 48,11 (1971).

5 R. P. Zhentevskaya, Neurotrophic Regulation of the Plastic Activity of Muscular Tissue (Nauka, Moscow,) Russian.

${ }^{6}$ B. M. Carison and E. Gutmann, unpublished observations.

7 A. J. Buller, J. C. Eccles and R. M. Eccles, J. Physiol., Lond. $150,417(1960)$.

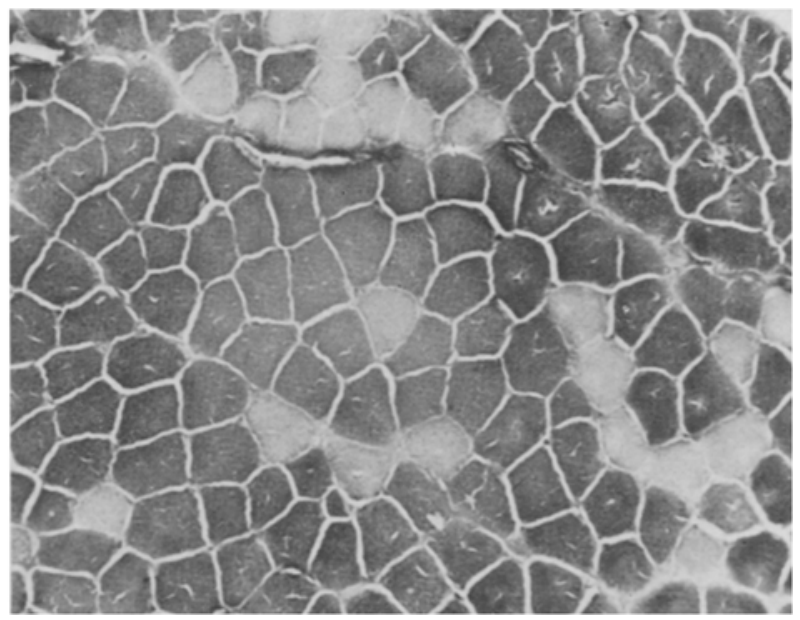

Fig. 2. 60-day extensor digitorum longus transplant stained for ATPase activity. Dark fibres with high amounts of ATPase activity may now be clearly distinguished from light fibres with less activity. 
Table I. Contraction times (in msec) of free grafts of normal and 14 day denervated EDL (fast) and SOL (slow) muscles

Age (days)

(N) Normal EDL

(N) Denervated EDL

(N) Normal SOL

(N) Denervated SOL

(5) $20.54 \pm 0.33$

(4) $19.85+0.35$

(5) $24.00 \pm 0.60$

(4) $21.28 \pm 0.92$

(5) $14.90 \div 0.91$

(4) $11.97 \pm 0.30$

$\begin{array}{ll} & - \\ \text { (5) } 34.04 \pm 2.50 \\ \text { (3) } 33.66 \pm 2.03 \\ \text { (3) } 27.33 \pm 0.75 \\ \text { (4) } 26.60 \pm 0.31 \\ \text { (4) } 19.15 \pm 2.88 \\ \text { (4) } 28.00 \pm 1.80\end{array}$

$\begin{array}{ll}\text { (4) } & 32.65 \pm 1.31 \\ \text { (6) } & 30.23 \pm 1.97 \\ \text { (5) } & 31.62 \pm 1.04 \\ \text { (7) } 29.81 \pm 0.68 \\ \text { (3) } 22.70 \pm 2.20 \\ \text { (3) } 22.00 \pm 1.00 \\ \text { (3) } 28.83 \pm 3.24\end{array}$

corresponding muscle of the contralateral leg. The transplanted muscle was then sutured at either end to the appropriate tendon stumps of origin and insertion. This technique does not cause any mechanical damage to the muscle fibres of the transplant, but the freshly transplanted muscle has no connection to a blood supply or to nerves. There were 2 experimental groups in this series. In one, normal muscles were transplanted and in the other, the muscles were denervated for 14 days prior to transplantatation.

For the cross-transplantation experiments all muscles were denervated for 14 days before grafting into the contralateral normal limb. Previously denervated SOL muscles were grafted in place of the contralateral EDL, and EDL muscles were grafted in place of the SOL.

At various periods up to 90 days after the operation, grafted muscles were removed for histological, histochemical and physiological analysis. Contractile responses were measured following direct electrical stimulation in vitro as described earlier ${ }^{8}$. After determination of contractile properties, the grafts were either fixed in Bouin's or frozen in liquid-nitrogen and tested histochemically for phosphorylase (Ph), succinic dehydrogenase (SDH) and myosin adenosine triphosphatase (ATPase).

Results. Grafts of normal or denervated muscles. Although both normal and denervated muscles respond to free

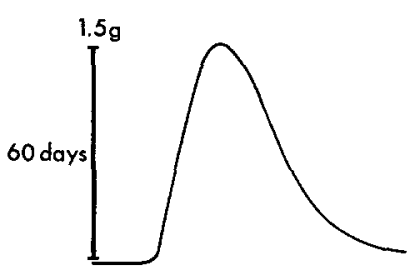

$\mathrm{EDL} \rightarrow \mathrm{SOL}$
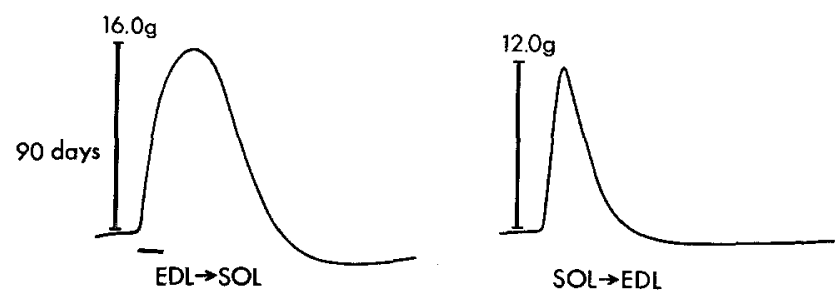

Fig. 3. Representative contraction curves for cross-transplanted muscles at 60 and 90 days. Extensor digitorum longus in place of the soleus on the left side and soleus in place of the extensor digitorum longus on the right. The horizontal line beneath the lower left hand curve represents $10 \mathrm{msec}$. grafting by degeneration of the original muscle fibres followed by the regeneration of new ones, there are some pronounced differences between the 2 types of grafts during the first 10-14 days after grafting. The overall histological reaction or grafted normal muscles is similar to that seen in a minced muscle ${ }^{9}$. The peripheral muscle fibres degenerate first and are replaced by regenerating muscle fibres. As the wave of degeneration of old muscle, followed by the regeneration of new muscle fibres, sweeps toward the center of the graft, a radial gradient of regeneration is set up, with the most mature regenerating muscle fibres being at the periphery. At the very periphery, occasional fibres of the original muscle remain histologically intact throughout the post-transplantation period.

In denervated grafts, particularly the SOL, a clear-cut radial gradient of degeneration and regeneration is not set up. Instead, the grafts are characterized by the very swift appearance of regenerating muscle fibres throughout the graft, leading to the establishment of a fairly uniform population of thin cross-striated muscle fibres as early as 5 days after transplantation in the SOL. In denervated grafts, larger numbers of thin, original muscle fibres persist at the periphery of the grafts. After 2-3 weeks there is little histological difference between grafts of normal and previously denervated muscle.

The pattern of development of contractile properties was complex. In grafts of normal SOL and EDL, little or no contractile activity was noted during the first 2-4 days after transplantation. At 4 days the muscles contracted weakly, the contraction time (time to peak $=$ CT) being slightly prolonged against the normal EDL (CT of normal EDL - $11.60 \pm 0.60 \mathrm{msec}$ ) because of the 4-day denervation period after transplantation. In the EDI, the CT's became slower during the 2nd and 3rd weeks and then progressively speeded up to normal values (Table I). The SOL grafts demonstrated a temporary shortening of the $\mathrm{CT}$, followed at 90 days by a prolongation. This is apparently a reflection of the normal tendency of the SOL to become faster and then slow down again as the young animal matures ${ }^{10,11}$.

In contrast to grafts of normal muscle, the contractility of 14-day denervated grafts persisted during the early postoperative days. In the EDL, the early CT's were slow, resembling those of 2 -week denervated muscle. The CT's

${ }^{8}$ B. M. Carlson and E. Gutmann, Expl Neurol. 36, 239 (1972). 9 B. M. CARLson, The Regeneration of Minced Muscles (S. Karger AG, Basel).

10 A. J. Buller, J. C. Eccles and R. M. Eccles, J. Physiol., Lond. $150,399(1960)$.

11 E. Gutmann and J. Melichna, Physiologia bohem. 21, 1 (1972). 
Table II. Contraction times (in msec) of cross-transplanted EDL (fast) and SOL (slow) muscles

\section{Days}

(N) SOL into EDL

(N) EDLinto SOL

\begin{tabular}{llll}
17 & (3) $24.13 \pm 0.76$ & (3) $23.30 \pm 0.30$ \\
30 & (4) $20.43 \pm 0.90$ & (5) $21.60 \pm 0.82$ \\
60 & (5) $13.90 \pm 0.66$ & (4) $20.97 \pm 0.92$ \\
90 & (6) $12.95 \pm 0.26$ & (4) $23.55 \pm 1.20$ \\
\hline
\end{tabular}

remained slow during the first 2 weeks after transplantation, after which time they gradually began to speed up to normal contraction times. Except for the ability to contract throughout the early postoperative days, denervated SOL transplants did not differ from normal grafts to as great an extent as the EDL.

Histochemically demonstrable $\mathrm{Ph}$ activity in 2-4 day grafts of normal muscles was absent except for a narrow rim of scattered fibres at the periphery. Early denervated grafts were also largely inactive in the center, but consistently demonstrated a broader rim of enzymatically active fibres. As newly regenerating muscle fibres appeared, an increasing degree of overall enzymatic activity returned to all grafts. At 30 days, the muscle fibres still showed a uniform pattern with respect to ATPase activity (Figure 1), but later a mixed pattern of fibre types developed (Figure 2).

Cross-transplanted grafts. Cross-transplanting of both the EDL and SOL muscles produced major changes in contractile and histochemical properties. SOL muscles grafted into the bed of the EDL demonstrated a complete conversion of contractile properties from slow to fast muscles (Figure 3 and Table II). Cross-transplanted EDL muscles slowed down considerably, but never became as slow as the normal SOL.

The histochemical properties of the cross-transplanted muscles mirrored the changes in contractile properties. After cross-transplantation, the fibre type pattern (ATPase) of the SOL is converted to that of the EDL, and the cross-transplanted EDL resembles the normal SOL.

Discussion. Free grafts of both normal and previously denervated SOL and EDL muscles are successful in young rats. In both cases, the reason for the success is the replacement of original muscle fibres in the grafts by newly regenerating muscle. The major differences between the 2 types of grafts are seen during the first week after transplantation, and they seem to stem from the very rapid rate at which new muscle fibres appear in denervated grafts. The reasons for this are still obscure, but may be related to previous reports of increased numbers of mononuclear cells appearing beneath the basement membranes of denervated muscle fibres ${ }^{12,13}$. These cells (whether true satellite cells or not cannot be stated at this time) could be myoblastic, and their presence in denervated muscle would then give it a head start in the regenerative process as compared with normal grafts. This phenomenon might also account, at least in part, for the success of grafts of denervated large muscle as opposed to the failures of large grafts of normal muscles.

The nature of the early contractions of freely grafted muscles appears to be due to two contributing phenomena. One is the persistence of a small population of original muscle fibres which have been denervated by the act of transplantation itself. This causes a slight prolongation in their CT. The other is the later superimposition of the contractions of the regenerating muscle fibres onto the contractions of the remaining mature muscle fibres in the graft. During the first week in normal muscle, and during the first 4 days in denervated muscle, any contractions are due entirely to the few persisting original muscle fibres along the periphery of the graft. Then during the next 2 weeks, newly regenerating fibres begin to make a contribution. In fast muscles, at least, they contract much more slowly than normal muscle fibres ${ }^{8}$. At later periods the regenerating muscle fibres have matured to the point where there is little difference between their CT's and those of normal muscle.

The absence of contractile activity in early grafts of normal muscle contrasts sharply to its persistence in grafts of denervated muscle. This is correlated with a pronounced temporary decrease in the resting membrane potentials of superficial muscle fibres in grafts of the normal EDL during the first days after transplantation ${ }^{14}$ There is relatively little change in early grafts of denervated muscle. In addition, the histochemical observations reveal far fewer enzymatically active muscle fibres in early grafts of normal than in denervated muscles.

The cross-transplantation studies in free grafts demonstrate that regenerating muscle fibres are equally subject to the influence of their innervation as crossinnervated muscles which remain in their normal location. Conversion of the cross-transplanted SOL is virtually complete whereas the transformation of contractile properties is less marked in the cross-transplanted EDL. The incomplete conversion of the EDL during the experimental period might be due to the temporary developmental speeding that is seen in normal slow muscles ${ }^{10,11}$. Longer term observations would be needed to test for a later period of slowing down.

The method of free grafting of entire mammalian muscles has proven to be of considerable value in studies on the functional properties of regenerating muscle. With the recent application of the free grafting technique to human muscles, the method appears to offer further opportunities for application in clinical medicine.

Zusammentassung. Nachweis, dass ein frei transplantierter Rattenmuskel besser regeneriert, wenn er 14 Tage vor der Transplantation denerviert wurde.

\section{B. M. Carlson and E. Gutmann}

Department of Anatomy, University of Michigan, 4622 Medical Sciences II, Ann Arbor (Michigan 48104, USA), and Institute of Physiology, Czechoslovak Academy of Sciences, Praha 4-Krč (Czechoslovakia), 25 June 1974.

12 J. C. LEE, Expl Neurol. 12, 123 (1965).

13 A. Hess and S. Rosner, Am. J. Anat. 129, 21 (1970).

14 F. Vyskočil, B. Carison and E. Gutmann, Pflügers Arch. ges. Physiol. 344, 181 (1973). 